\title{
Erratum to: Pure Strategy Nash Equilibrium and Simultaneous-Move Games with Complete Information
}

\section{Erratum to: \\ Chapter 2 in: F. Munoz-Garcia and D. Toro-Gonzalez, Strategy and Game Theory, Springer Texts in Business and Economics, https://doi.org/10.1007/978-3-319-32963-5_2}

In the original version of the book, the post-publication corrections to update the equations in Chapter 2 have been incorporated. The erratum chapter and the book have been updated with the changes. 